\title{
Variation of the Vibration Profile of Piezoelectric Resonant Sensors with Different Electrode Conductivity at High Temperatures
}

\author{
Silja Schmidtchen, Denny Richter, Holger Fritze, \\ Clausthal University of Technology, \\ Institute of Energy Research and Physical Technologies, \\ Am Stollen 19B, 38640 Goslar \\ silja.schmidtchen@tu-clausthal.de
}

\begin{abstract}
:
Langasite based piezoelectric resonators coated with metal oxide films are used as gas sensors for high-temperature applications. The influence of changes in electrical and mechanical properties of those films on the vibration behavior of the resonator is investigated with a laser Doppler interferometer. For this purpose, two different electrode geometries are used, leading to a microbalance mode and a conductivity mode. Thereby, the shift of the resonance frequency caused by a change of the film mass as well as the modification of the vibration profile can be distinguished. Resonators used in the conductivity mode provide information about the oxygen partial pressure. By using both effects simultaneously, a higher gas selectivity of those sensors is achieved.
\end{abstract}

Key words: laser Doppler interferometer, langasite, high-temperature, piezoelectricity

\section{Introduction}

Nowadays research in the field of sensors has to take into account the increasing demands for efficient energy conversion in e. g. fuel cells or decentralized power plants. That requires, in particular, high sensitivity and selectivity for gases in combustion processes. Especially, the strong requirements for the materials stability and compatibility in high-temperature environments have to be fulfilled. In this context, piezoelectric gas sensors show several advantages compared to optical or resistive sensor systems, such as the option to perform in-situ measurements in harsh environments and to detect changes in the mechanical properties of sensor films. Therefore, the research is focused on sensors based on the high-temperature stable piezoelectric material langasite $\left(\mathrm{La}_{3} \mathrm{Ga}_{5} \mathrm{SiO}_{14}\right)$ which exhibits a high thermal and chemical stability. It shows no phase transition up to its melting point at $1470{ }^{\circ} \mathrm{C}$ and low damping up to about $1000{ }^{\circ} \mathrm{C}$ [1]-[2]. Langasite can be used as a piezoelectric transducer up to temperatures of at least $1000^{\circ} \mathrm{C}$, in contrast to common quartz resonators which operation limit is at about $450{ }^{\circ} \mathrm{C}$. In order to develop new technologies for miniaturization of sensor systems and preparation of sensor arrays, a detailed investigation of the sensor properties is necessary, including electrical and mechanical properties as well as the influence of the specific sensor design. To understand and optimize this effect, the correlation of the sensor film properties and the spatial distribution of the vibration amplitude of the resonator have to be determined. The latter is realized with a laser Doppler interferometer at high temperatures and reducing atmospheres.

\section{Sensor Principle}

Two different operation principles are possible for piezoelectric gas sensors (see Fig. 1). Both are based on langasite resonators coated with a gas sensitive metal oxide film. Operating the sensor in the microbalance mode [3], atmosphere induced changes in the mass of the applied film can be detected as a frequency shift. In the conductivity mode a change in the composition of the surrounding gas influences the conductivity of the film, which, on the other hand, affects the vibrational properties of the resonator. 


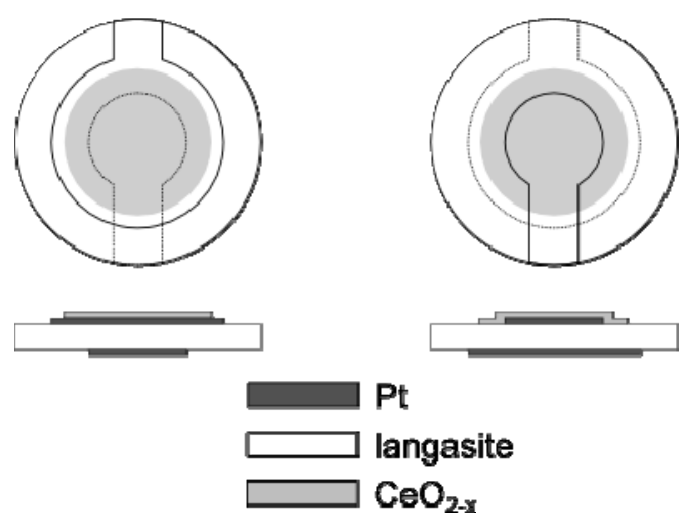

Fig. 1. Electrode layout for piezoelectric resonators operated in the microbalance mode (left) and the conductivity mode (right).

The vibration profile of a resonator and consequently the sensitivity, which is directly proportional to the vibration amplitude, strongly depend on the geometries of electrodes and sensor film. Thereby, the amplitude distribution of the displacement can be approximated using a Gaussian distribution with a maximum in the center of the electrodes [4]. The y-cut langasite resonators used here are excited to thickness shear mode vibrations with a resonance frequency governed by its thickness and the mass of electrodes and film. Outside the electrode area the resonance frequency would be higher due to the absent mass load. Therefore, the vibration amplitude exhibits a strong decay at the border of the electrode. In other words, the vibration energy is strongly localized in the electrode area (energy trapping).

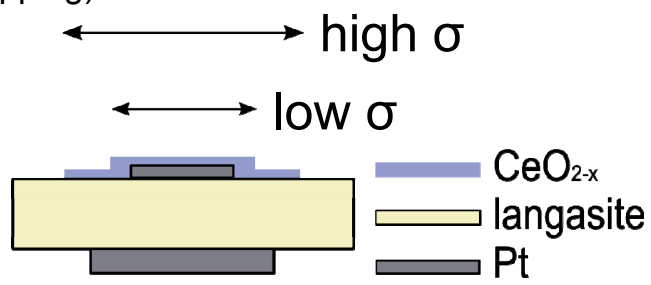

Fig. 2. Schematic drawing of a resonator with platinum electrodes and $\mathrm{CeO}_{2-x}$ film. Arrows indicate insulating film (low $\sigma$, small effective electrode diameter) and high conductivity (high $\sigma$, large effective electrode diameter).

The spatial distribution of the displacement amplitude and, thereby, the mass sensitivity depend on the size of the excited area of the resonator as shown schematically in Fig. 2. In other words, the extension of the vibration profile is determined by the diameter of the conductive electrodes. The vibration distribution of resonators with different electrode diameters is shown schematically in Fig. 3.

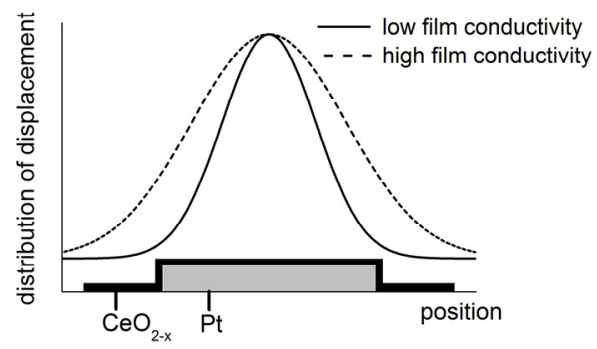

Fig. 3. Model of the change of the effective electrode area depending on the conductivity of the $\mathrm{CeO}_{2-x}$ sensor film.

\section{Experimental Setup}

To investigate the influence of the effective electrode diameter, keyhole shaped platinum electrodes with different diameter on front and backside of the resonator (Fig. 1) are applied. In addition, a metal oxide film $\left(\mathrm{CeO}_{2-\mathrm{x}}\right)$ is deposited on the smaller metal electrode and exceeds its size. Under reducing atmospheres the conductivity of the film increases, resulting in an enlargement of the effective electrode area.

The bulk acoustic wave resonator used is a $y$ cut langasite disc with $19 \mathrm{~mm}$ in diameter and a thickness of about $600 \mu \mathrm{m}$. The fundamental thickness shear mode exhibits a resonance frequency of $2.2 \mathrm{MHz}$. The larger electrode on the front side of the resonator, $10.3 \mathrm{~mm}$ in diameter, is screen printed to ensure a sufficiently rough surface since a diffuse scattering is required for the interferometer measurements. The thickness of this electrode is about $3 \mu \mathrm{m}$. The smaller electrode at the back side, $5.8 \mathrm{~mm}$ in diameter and $300 \mathrm{~nm}$ in thickness, is deposited by pulsed laser deposition. The $\mathrm{CeO}_{2-x}$ film is pulsed laser deposited with a thickness of $200 \mathrm{~nm}$. It is applied on the backside electrode, overlapping the platinum electrode with a diameter of $13.2 \mathrm{~mm}$ (Fig. 2).

The piezoelectric samples are mounted in an alumina sample holder in a gas tight tube furnace which enables operating temperatures up to $1000^{\circ} \mathrm{C}$. The interferometer is a single-point laser Doppler vibrometer (Polytec OFV 505), placed outside the furnace. The working distance between laser and sample is about $0.5 \mathrm{~m}$ with a minimum spot size of $18 \mu \mathrm{m}$. Local displacements below $1 \mathrm{~nm}$ are detectable [5]-[6]. Line scans or raster scans of the sample surface are realized by changing the position of the laser beam. Thereby, the laser beam is deflected with a mirror tilt system. The adjustment of the oxygen partial pressure is realized using a gas mixing system and an 
oxygen ion pump and measured with a zirconium oxide oxygen sensor. The oxygen partial pressure dependent conductivity of the $\mathrm{CeO}_{2-x}$ film is measured independently using an impedance analyzer Solartron 1260. Further, the bulk properties of the resonators such as the bulk resistance $R_{b}$ are investigated by impedance spectroscopy with the impedance analyzer mentioned above. The resonance properties are determined with a network analyzer HPE5100A [7].

\section{Measurements and Results \\ Electrical properties of $\mathrm{CeO}_{2-x}$ :}

The oxygen partial pressure dependent conductivity of a $\mathrm{CeO}_{2-x}$ film is determined at temperatures up to $600{ }^{\circ} \mathrm{C}$ [6]. As shown in Fig. 4 , the conductivity increases with decreasing oxygen partial pressure with $\sigma \sim p^{m}{ }_{02}, m=-1 / 10$ for partial pressures below $10^{-12}$ bar and $\mathrm{m}=$ $-1 / 6$ for higher pressures.

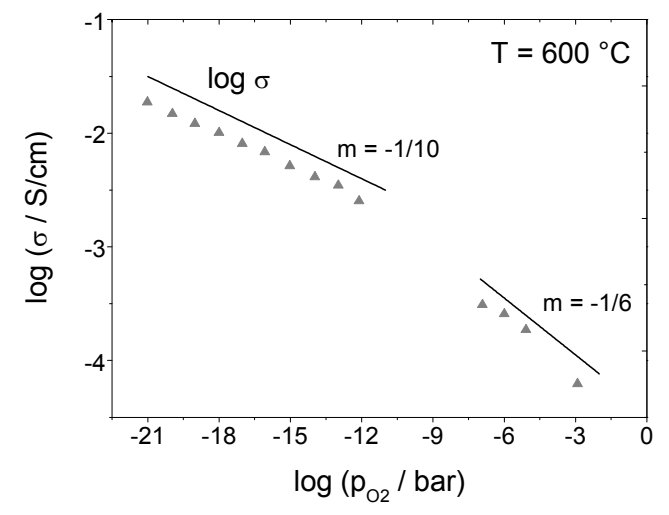

Fig. 4. Oxygen partial pressure depending conductivity of the $\mathrm{CeO}_{2-x}$ film.

\section{Impedance measurements:}

As described earlier in [7] measurements of the electrical properties of a resonator with an impedance analyzer demonstrate the broadening of the active resonator area indirectly. The electrical properties of a resonator with a similar electrode design and metal oxide film as described above are shown in Fig. 5 as a function of the oxygen partial pressure.

Since the resonator material langasite does not show changes in conductivity at $580{ }^{\circ} \mathrm{C}$ if the oxygen partial pressure is lowered down to $10^{-21}$ bar, changes in electrical properties of an entire device must be related to the sensor film. In particular, the extent of the electrode must be regarded. Approximating the electrical properties of the resonator by the extended Butterworth-van-Dyke equivalent circuit [2], the bulk and motional properties denoted by the index $\mathrm{b}$ and $\mathrm{m}$, respectively, are determined. Those properties are clearly depending on the electrode area, as can be seen in Fig. 5 . Capacitances $C_{m}$ and $C_{b}$ are proportional to the electrode area $A$ while the resistance $R_{b}$ is inversely proportional to $A$.

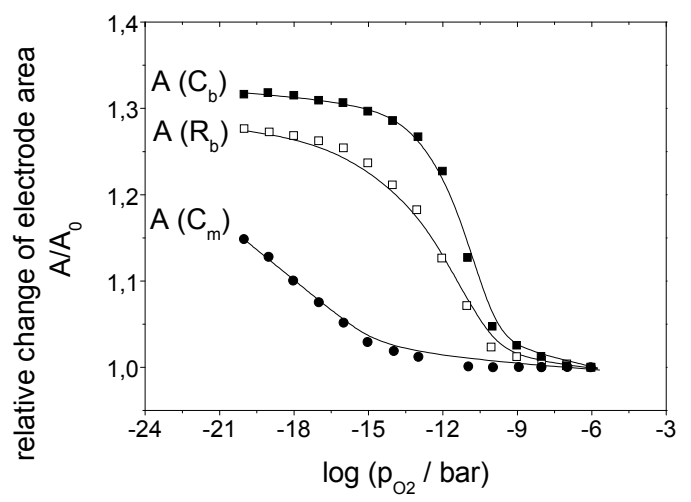

Fig. 5. Relative changes of the parameters of the electrical equivalent circuit depending on the oxygen partial pressure with $A_{0}$ denoting the area of the platinum electrode.

The increase in the conductivity of $\mathrm{CeO}_{2-x}$ with decreasing oxygen partial pressure can be directly related to the changes of the Butterworth-van-Dyke equivalent circuit parameters as done in detail in [7].

Vibration profile:

Vibration amplitudes of the fundamental mode and the overtone are measured at room temperature with line scans with step sizes of about $250 \mu \mathrm{m}$ (see Fig. 6). The fundamental mode exhibits a significantly wider profile than the overtone. The variation of the Gaussian profile of the vibration amplitude is measured at different oxygen partial pressures from $10^{-4}$ to $10^{-20}$ bar at temperatures up to $580^{\circ} \mathrm{C}$.

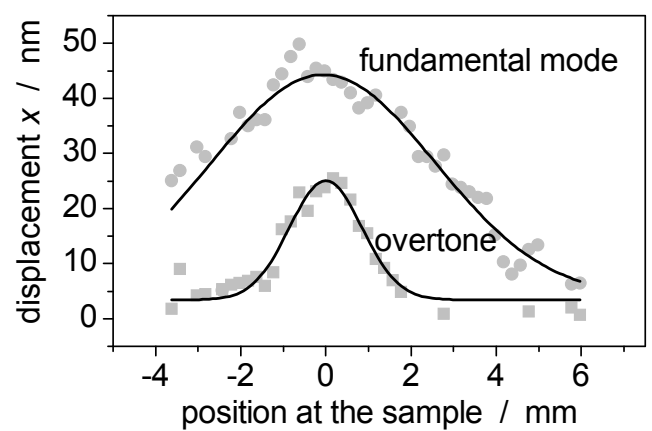

Fig. 6. Spatial distribution of the displacement of a resonator excited with resonance frequencies of fundamental mode (2 $\mathrm{MHz})$ and overtone $(6 \mathrm{MHz})$ at room temperature.

For further discussions the results are fitted with a Gaussian function and normalized to unity. 
The resulting broadening of the vibration profile corresponding to the enlargement of the effective electrode area is shown in Fig. 7.

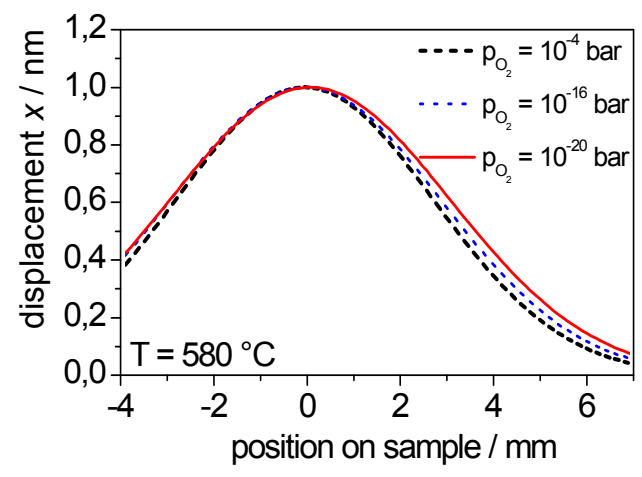

Fig. 7. Vibration profile of a resonator with a $\mathrm{CeO}_{2-x}$ film at $580{ }^{\circ} \mathrm{C}$ and three different oxygen partial pressures.

By calculating the full width of half maximum it becomes obvious that the reduction of the oxygen partial pressure causes an increase of the effective electrode diameter by factor 1.09 .

Comparing these results with those from the electrical measurements, very similar results are obtained. For example, the increase of the motional capacity $\mathrm{C}_{\mathrm{m}}$ corresponds to an increase of the effective electrode diameter by a factor 1.07, which is calculated from the square root of $A / A_{0}$ in Fig. 5 .

\section{Raster scanning:}

In order to achieve more detailed information about the spatial distribution of the mechanical displacement, a raster scan is realized at room temperature with $100 \times 100$ data points. In Fig. 8 the amplitude distribution is shown.

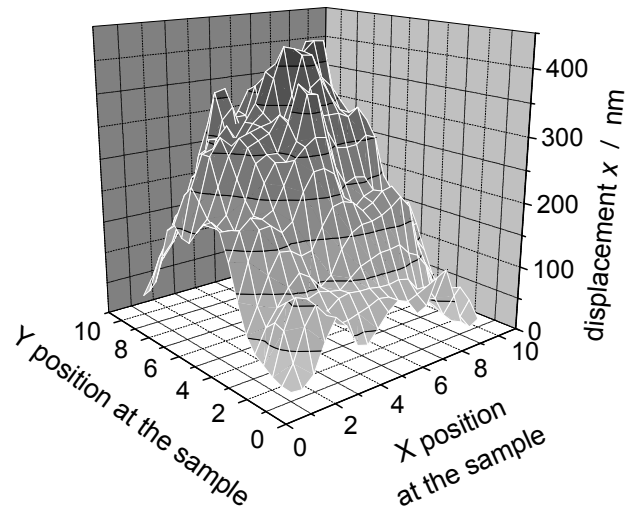

Fig. 8. Raster scan of a ceria deposited langasite resonator in $3 D$ visualization.

The maximum amplitude is located in the center of the resonator and decays towards the border of the excited resonator area. Deviations from the expected Gaussian profile can be assigned to measurements uncertainties and an irregular thickness of the screen printed electrode.

\section{Conclusion}

The spatial distribution of the vibration amplitude of a langasite resonator could be measured with a laser Doppler interferometer in a line scan mode at temperatures up to $580^{\circ} \mathrm{C}$. The fundamental mode and the first overtone are compared. The overtones exhibit significantly narrower vibration profiles and smaller amplitudes than the fundamental mode. This has to be taken into account for the evaluation of the mass sensitivity of a resonator operated at an overtone. Furthermore, the influence of the electrode diameter on the vibration profile can be determined with this measurement method. An enlargement of the electrode area causes a broadening of the amplitude distribution. A raster scan of the vibration profile is made to specify the spatial distribution. The results meet the expections with regard to the maximum amplitude found to be in the center of the electrode and decaying amplitude towards the boundary.

\section{Acknowledgements}

Financial support from the German Research Foundation (DFG) and the Energy Research Centre of Lower Saxony made this work possible.

\section{References}

[1] K. Shimamura, H. Takeda, T. Kohno and T. Fukuda, J. Cryst. Growth 163, 388-392, (1996), doi: 10.1016/0022-0248(95)01002-5

[2] H. Fritze, High-temperature bulk acoustic wave sensors, Meas. Sci. Technol. 22 (2011) 12002 (28pp), doi:10.1088/09570233/22/1/012002

[3] G. Sauerbrey, Zeitschrift für Physik $A$ Hadrons and Nuclei 155, 206-222 (1959); doi:10.1007/BF01337937

[4] B. A. Martin, H. E. Hager, J. Appl. Phys. 65(7), 2630-2635 (1989); doi:10.1063/1.342772

[5] S. Schmidtchen, J. Sauerwald, H. Xia, H. Fritze, AIP Conference Proceedings 1253(1), 164-170 (2010); doi:10.1063/1.3455454

[6] D. Richter, Selektiver Gassensor auf Basis hochtemperaturstabiler piezoelektrischer Langasitresonatoren, Dissertation (2011) TU Clausthal

[7] H. Fritze, D. Richter, and H. Tuller, Sensors \& Actuators: B. Chemical, 111:112, (2005), doi.org/10.1016/j.snb.2005 